\title{
Aprendizaje de la composición de simetrías axiales apoyados con el software de geometría dinámica GeoGebra ${ }^{\circledR}$
}

\section{Learning the composition of axial symmetries supported with GeoGebra ${ }^{\circledR}$ dynamic geometry software}

Jhonatan Andrés Robayo-Buitrago ${ }^{\mathrm{a}}$, Cesar Andrés Quezada-Alfonso ${ }^{\mathrm{b}}$, Oscar Jardey Suarez $^{\mathrm{c}}$

\begin{abstract}
aMagister en Educación, jarobayob@correo.udistrital.edu.co, https://orcid.org/0000-0003-4113-5983, Colegio Refous, Bogotá, Colombia ${ }^{b}$ Magister en Educación, caquezadaa@correo.udistrital.edu.co, https://orcid.org/0000-0002-8551-9586, Colegio Refous, Bogotá, Colombia 'Doctor en Educación, oscar.jardey.suarez@gmail.com, https://orcid.org/0000-0001-8780-595X, Universidad Distrital Francisco José de Caldas, Bogotá, Colombia
\end{abstract}

Forma de citar: Robayo-Buitrago, J. A.. Quezada-Alfonso, C. A. ., \& Suárez, O. J. . (2021). Aprendizaje de la composición de simetrías axiales apoyados con el software de geometría dinámica GeoGebra ${ }^{\circledR}$. Eco Matemático, 12 (2), 25-36

Recibido: 10 de marzo de 2021

Aceptado: 30 de abril de 2021

\section{Palabras clave}

Aprendizaje Basado

en Proyectos

Colaborativos,

Educación en medio

de la pandemia, simetrías axiales, educación presencial remota.
Resumen:El objetivo de la investigación es comparar el aprendizaje en la composición de simetrías axiales apoyado con GeoGebra en dos grupos de estudiantes, uno a través de la enseñanza que habitualmente se trabaja en la institución y otro con el Aprendizaje Basado en Proyectos Colaborativos (ABPC), en medio de la pandemia debido a la COVID-19. El enfoque metodológico de la investigación es principalmente cuantitativo, se apoya en las pruebas de hipótesis U-Mann Whitney y Wilcoxon. Las actividades se caracterizaron por ser sincrónicas (Plataforma Teams de Microsoft ${ }^{\circledR}$ ) y asincrónicas (OneDrive ${ }^{\circledR}$, GeoGebra y Teams). La Institución Educativa tiene énfasis en matemáticas y está ubicada en un municipio cercano a la ciudad de Bogotá-Colombia. Los resultados muestran ganancia, estadísticamente significativa, en ambos grupos, en el aprendizaje de la composición de simetrías axiales; sin embargo, el grupo que trabajó con el ABPC, presenta una mayor media en su aprendizaje. Entre las principales conclusiones está que organizar el trabajo, desde la actividad docente, para ser desarrollado en grupo a través de estrategias como el ABPC apoyado con GeoGebra, puede tener potencialmente, una mayor ganancia, en relación con el logro de aprendizaje de la geometría.

*Autor para correspondencia jarobayob@correo.udistrital.edu.co

Doi: https://doi.org/10.22463/17948231.3185 


\section{Keywords}

Learning Based on Collaborative Projects, Education in the midst of the pandemic, axial symmetries, remote face-to-face education.

\begin{abstract}
The objective of the research is to compare the learning in the composition of axial symmetries supported with GeoGebra in two groups of students, one through the teaching that is usually worked in the institution and the other with the Learning Based on Collaborative Projects (ABPC), in the midst of the pandemic due to COVID-19. The methodological approach of the research is mainly quantitative, it is supported by the U-Mann Whitney and Wilcoxon hypothesis tests. The activities were characterized by being synchronous (Microsoft ${ }^{\circledR}$ Teams Platform) and asynchronous (OneDrive ${ }^{\circledR}$, GeoGebra and Teams). The Educational Institution has an emphasis on mathematics and is located in a municipality near the city of Bogotá - Colombia. The results show a statistically significant gain in both groups in learning the composition of axial symmetries; However, the group that worked with the ABPC presented a higher average in their learning. Among the main conclusions is that organizing the work, from the teaching activity, to be developed in a group through strategies such as the ABPC supported with GeoGebra, can potentially have a greater gain, in relation to the achievement of learning geometry.
\end{abstract}

\section{Introducción}

La geometría dinámica se configura en los estudiantes, como una mediación en la construcción de nuevos esquemas (Sandoval-Cáceres, 2009), en el que el uso del software, como GeoGebra o Cabri, posibilita la construcción de diversos ambientes (Rubio-Pizzorno y Montiel-Espinoza, 2017) en pro de resolver problemas geométricos (Iglesias-Inojosa y Ortiz-Buitrago, 2019), en general posibilitando rutas de enseñanza-aprendizaje de la geometría (García y López, 2008), como el caso reportado por Sua-Florez y Camargo-Uribe (2019), en el que jóvenes de grado noveno establecen sinergia entre la geometría dinámica y el razonamiento científico.

La institución educativa (IE) en la que se desarrolla la investigación, está ubicada en un municipio cercano a la ciudad de Bogotá Colombia. La IE tiene énfasis en matemáticas y el proceso de enseñanza - aprendizaje se caracteriza, habitualmente, por realizar las construcciones mediante el uso de regla y compás; actualmente por la pandemia, se ha incorporado el uso de las Tecnologías de la Información y la Comunicación (TIC) (Gómez y Ramírez, 2016) disponibles en la internet, como GeoGebra para el caso de la geometría. Una de las exigencias cognitivas de la institución corresponde a que los estudiantes realicen predicciones de transformaciones de simetrías axiales; este tópico está abordado de manera general en los lineamientos y estándares matemáticos para el contexto colombiano, como parte del desarrollo del pensamiento espacial (Ministerio de Educación Nacional, 1998).

En el marco de la enseñanza-aprendizaje, los proyectos colaborativos motivan la colaboración y participación colectiva de los estudiantes en las diferentes actividades que se propongan, en el que el rol del docente pasa a establecer el alcance, así como las intenciones propias de cada una de las actividades que se proponen en el acto educativo (Maldonado, 2008; Morera, et al. 2012).

En cuanto a la geometría dinámica, soportada en Software, existen estudios como los de Arbain y Shukor (2015), Villamizar, Rincón y Vergel (2017) y Fajardo y Larios (2019), en los cuales se describe que la enseñanza-aprendizaje de las matemáticas, no debe centrarse solamente un énfasis teórico, sino que debe pensarse desde los diferentes estilos de aprendizaje y en cómo motivar dichos estilos, adicionalmente señalan la necesidad de promover el pensamiento crítico con el apoyo de las TIC, 
por ejemplo, GeoGebra. Así mismo, en cuanto al razonamiento geométrico, se encuentra que éste se ve favorecido si se tienen en cuenta aspectos como: una secuencia de situaciones de construcción geométrica relacionadas entre sí, en las que se ponga a prueba la deducción de los estudiantes, el aprovechamiento de las facilidades que proporciona la geometría dinámica acompañada de la resolución de problemas y la mediación semiótica del profesor (Mariotti, 2009), quien debe estar pendiente de las construcciones de los estudiantes y de proponer situaciones en las cuales, a partir del discurso, se evalúa el significado matemático-geométrico de los signos o elementos puestos en juego (Camargo \& Samper, 2013).

\section{Fundamentos Teóricos}

Para Badia y García (2006) el Aprendizaje Basado en Proyectos Colaborativo (ABPC), se entiende como una propuesta didáctica que parte de proyectos situados en la realidad próxima, propuestos por o a los estudiantes, para que sean desarrollados en equipo de trabajo, promoviendo la colaboración entre pares. Las TIC como soporte del ABPC, permiten la interacción bidireccional entre el profesor, el estudiante y el conocimiento, tal como se indica en la Figura 1. Mesa, Fernández y Duarte (2018) consideran siete etapas en el planteamiento, diseño desarrollo del proyecto basado en ABPC: (1) selección de grupos de trabajo, (2) planteamiento de preguntas para orientar el proceso, (3) indagaciones previas para la construcción del proyecto, (4) planificación del proyecto, (5) organización y análisis de información, (6) descripción y presentación de resultados y (7) informe final y cierre.

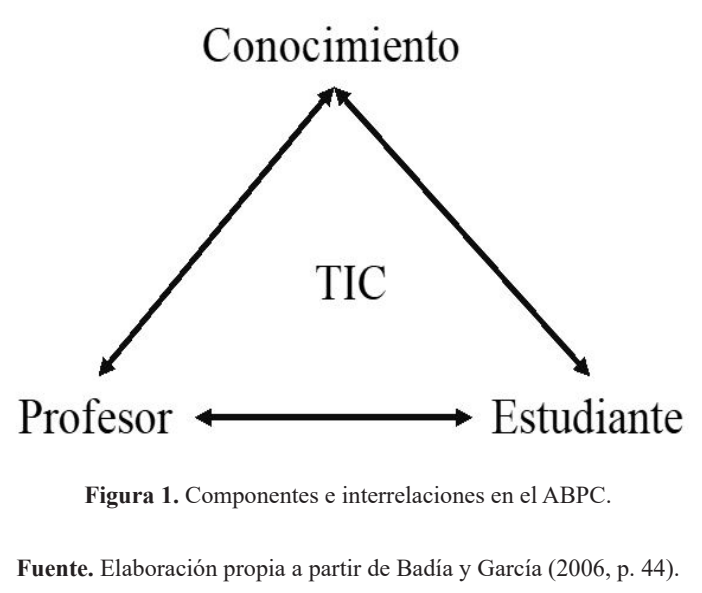

En el ABPC los estudiantes trabajan colaborativamente, deben mediar con los objetivos a realizar, la representación de la situación planteada, al igual que el contenido conceptual y procesos involucrados. En ese proceso de intercambio de argumentos y reconocimiento de significados, que pueden cambiar, mejorar o ampliarse, toma gran importancia las interacciones entre los mismos estudiantes, al igual que las interacciones con el profesor.

El estudio de la geometría dinámica promueve en los estudiantes operaciones cognitivas en la reconstrucción de conocimientos que interactúan con sus diferentes sentidos y con la realidad circundante (Ministerio de Educación Nacional, 1998), en la que se identifican diversos modelos, objetos que potencialmente pueden ser utilizados como mediación (Bartolini-Bussi y Mariotti, 2008, Godino y Ruíz, 2003) u objeto de conocimiento en el proceso de enseñanza-aprendizaje de la geometría; entendiendo los elementos de mediación como un componente activo e inseparable de las diversas acciones en dicho proceso (Moreno, 2014).

Las TIC como elemento mediador en la construcción de conocimiento matemático geométrico en el estudiante (Villamizar, 2020), son un elemento que potencia en el aula de clase, el diseño e implementación de ambientes de aprendizaje. 
GeoGebra, como una expresión de las TIC, es un software que posibilita rutas de aprendizaje que facilitan operaciones cognitivas, como la modificación y concebir objetos geométricos (RuizVahos, et. al, 2013). Lo anterior, está en consonancia con la National Council of Teachers of Mathematics - NCTM (2000), quien plantea que efectivamente las TIC deben contribuir en favorecer el aprendizaje de las matemáticas.

En general, las TIC brindan beneficios a la educación, en cuanto permiten transformar las actuaciones de sus protagonistas; a los profesores les ha ayudado en sus prácticas educativas, posibilitando que los estudiantes amplíen la perspectiva de los elementos con los que se relacionan, ampliando las alternativas en los procesos de enseñanzaaprendizaje. En tiempo de pandemia debido a la COVID-19, ha soportado las interacciones entre y profesor en el rol de tutor, el estudiante y todas las dinámicas que subyacen a la enseñanza- aprendizaje (Suárez, Molina-Vásquez, Orjuela y Lizarazo, 2020) en los diversos entornos educativos forzados por la COVID-19.

\section{Método}

\section{Enfoque}

El enfoque de esta investigación es principalmente cuantitativo con un diseño cuasiexperimental(Guba y Lincoln, 1990; McMillan y Schumacher, 2005; Hernández-Sampieri, et. al 2018).

\begin{tabular}{cccc}
\hline G1 & 01 & X1 & 02 \\
\hline G2 & 01 & X2 & 02 \\
\hline
\end{tabular}

Figura 2. Diseño del cuasiexperimento.

Fuente. Elaboración propia

\section{Diseño Metodológico}

La actividad propuesta se desarrolló en dos grupos de estudiantes, con el diseño cuasi experimental mostrado en la Figura 2, en el que:

- G1. Grupo de estudiantes de grado octavo 1, denominado "grupo enseñanza Habitual".

- G2. Grupo de estudiantes de grado octavo 2, denominado "Grupo ABPC".

- O1. Pretest.

- X1. Secuencia didáctica basada en las características habituales de la Institución Educativa, apoyada con Geogebra.

- X2. Secuencia didáctica basada en el ABPC, apoyada con el Geogebra y demás características habituales de la Institución Educativa.

\section{- O2. Postest.}

En el diseño se tuvo presente que los grupos de estudiantes fueran equiparables, el pretest y el postest se aplicaron a ambos grupos y la secuencia de didáctica también fue la misma en cuanto a contenidos, incorporación de las TIC, en especial de GeoGebra, con un proyecto como dinámica de trabajo y las demás actividades propias institucionales; el único cambio es que para el grupo G2 se incorporó el ABPC como una exigencia de trabajo.

\section{Participantes}

Los estudiantes que se vincularon al proceso, con la previa autorización institucional y de sus padres, cursan octavo grado de educación básica; su organización fue por conveniencia toda vez que se está sujeto a la organización institucional. Cada grupo está conformado por 27 jóvenes, cuyas edades oscilan entre 13 y 16 años, quienes se vincularon al proceso a través de los servicios 
de la Internet, en la modalidad forzada debido a la COVID-19. Tecnológicamente, las comunicaciones se dieron a través de la plataforma Teams de Microsoft ${ }^{\circledR}$, que posibilita encuentros en línea sincrónicos y asincrónicos, así como posibilidades de almacenamiento a través de OneDrive ${ }^{\circledR}$.

\section{Instrumentos}

En la investigación se utilizaron tres instrumentos, los cuales se describen a continuación.

Pretest y Postest. En éstos se indaga el nivel de conocimiento que presentaban los estudiantes de cada grupo frente al reconocimiento de algunos aspectos geométricos. Las secciones son: reconocimiento de transformaciones (observación del conjunto preimagen y el conjunto imagen), uso del software GeoGebra como herramienta de construcción (procesos de construcción de la imagen de un conjunto de puntos, por una transformación $\mathrm{y}$ por el seguimiento de dos transformaciones) y el carácter predictivo en la composición de transformaciones (prever la composición resultante). Las transformaciones a las que se hace referencia son traslación, simetría central y simetría ortogonal.

Secuencia Didáctica basada en el ABPC. Esta se estructuró en dos unidades, la primera buscaba generar el estudio entorno a los contenidos matemáticos desde la exploración, para que sirviera como recurso en el desarrollo del proyecto. La segunda unidad estuvo centrada en la elaboración del proyecto propuesto a cada uno de los grupos de trabajo. El proyecto y las actividades se propusieron en el marco del arte con los teselados, dado que algunos de ellos se pueden construir por movimientos de traslación, rotación y reflexión, los cuales corresponden a los contenidos temáticos del grado, en particular, la composición de dichos movimientos.

La secuencia didáctica se desarrolló en 12 sesiones. (1) Pretest, (2) planteamiento del proyecto,
(3 y 4) composición de simetrías de ejes paralelos, (5 y 6) composición de dos simetrías de ejes secantes, (7) composición de tres simetrías de ejes paralelos, (8) composición de tres simetrías de ejes, $(9,10 \mathrm{y}$ 11) desarrollo y presentación del proyecto y (12) Postest. Las sesiones 1, 9, 10, 11 y 12 se desarrollaron en encuentros sincrónicos, en tanto que las sesiones 2 a 8 se desarrollaron asincrónicamente.

Para el diseño de los instrumentos fue necesario realizar una reflexión, tanto del objetivo de la investigación, así como del objetivo del proceso enseñanza-aprendizaje de la composición de simetrías axiales, en el marco de la IE, dando especial atención al carácter predictivo de las situaciones geométricas.

Los instrumentos tuvieron un proceso de validación de contenidos por parte de pares (expertos, profesores activos que enseñan matemáticas en este nivel, profesores magister en el área de la educación matemática o afín, así como profesores Magister en el área la tecnología o afín), y una prueba piloto (con estudiantes del mismo nivel).

\section{Hipótesis}

Las hipótesis que direccionaron el presente estudio de investigación son:

- H1. Muestras interrelacionadas (Pre test). El promedio de puntuaciones en el pre test del grupo G1, no tiene diferencias significativas con las obtenidas por el grupo G2.

- H2. Muestras interrelacionadas (Pos test). E1 promedio de las puntuaciones en la prueba pos test en los estudiantes del grupo G2, tiene diferencias significativas con las puntuaciones de estudiantes del grupo G1.

- H3. Muestras relacionadas. No existe diferencia entre las puntuaciones pretest y postest para el grupo G1. 
- H4. Muestras relacionadas. No existe diferencia entre las puntuaciones pretest y postest para el grupo G2.

A partir de las anteriores hipótesis se pretende, en primera instancia, identificar que entre el grupo G1 y el grupo G2 no existen diferencias significativas al inicio del proceso y forma que da validez al cuasi experimento. De otro lado, se pretende establecer para cada uno de los grupos, G1 y G2, si existen ganancias en el aprendizaje en las simetrías axiales. Finalmente establecer si hay diferencias significativas entre los grupos G1 y G2, en cuanto al aprendizaje de las simetrías axiales.

\section{Resultados y Discusión}

\section{Diseño de instrumentos}

Para la validación de los diferentes instrumentos se utilizó el coeficiente Aiken (V), el cual ha sido utilizado con éxito en algunas investigaciones (Boluarte-Carbajal y Tamari, 2017; Martin-Romera y Molina-Ruiz, 2017. La V de Aiken se calculó con la ecuación (1), para cada categoría. Donde $\mathrm{S}$ es la tu suma de los valores asignados por cada uno de los jueces en cada categoría, $n$ es el número de jueces, c es el número de la escala de valoración (para la presente investigación fue de 1 a 5 , en la que 1 es no es pertinente y 5 es totalmente pertinente).

$$
V=\frac{S}{(n(c-1))}
$$

En la Tabla I se encuentran los valores obtenidos en la validación de contenidos para el pretest y postest. Se observa que la valoración está entre 0.64 y 0.80 . Teniendo en cuenta que la menor valoración fue la de indicaciones claras, se decidió que en las sesiones del pretest y el postest, el equipo docente estaría permanentemente atendiendo a los estudiantes en las diferentes inquietudes, técnicas o de otra índole, que pudieran surgir. No se registraron inconvenientes en la aplicación de los instrumentos.

Tabla I. Resultados de la Validación de Contenido de Pretest y Postest

\begin{tabular}{llc}
\hline No. & \multicolumn{1}{c}{ Categoría } & V de Aiken \\
\hline 1 & Pertinencia frente al contenido geométrico. & 0,80 \\
2 & Coherencia con los objetivos de diseño. & 0,72 \\
3 & Organización conceptual. & 0,76 \\
4 & Usos de la herramienta GeoGebra. & 0,76 \\
5 & Lenguaje matemático. & 0,68 \\
6 & Indicaciones claras. & 0,64 \\
7 & Coherencia con los objetivos de investigación. & 0,80 \\
8 & Pertinencia con la pregunta de investigación. & 0,80 \\
& Promedio. & 0,75 \\
\hline
\end{tabular}

Fuente. Elaboración propia

En relación con la secuencia didáctica, el proceso de validación de contenido que se siguió fue igual que para el pretest y el postest. Los resultados se encuentran en la Tabla II, en la que se logra identificar que, para los jueces, existe una complejidad tanto en el uso del lenguaje, como en la pertinencia de los conceptos, en el entendido que está muy próximo al aspecto disciplinar de las matemáticas.
Dado que la IE tiene su énfasis en matemáticas, los aspectos mencionados no se constituyeron en obstáculos al momento de ser implementada la secuencia didáctica, sin embargo, si ésta fuese utilizada en una institución en la que no existe énfasis en matemáticas, necesariamente requiere de una adecuación a la población estudiantil con la cual va a ser usada. 
Tabla II. Resultados de la Validación de Contenido para la Secuencia Didáctica

\begin{tabular}{|c|c|c|}
\hline No. & Categoría & V de Aiken \\
\hline 1 & Evidencia de los principios del aprendizaje colaborativo. & 0,80 \\
\hline 2 & Coherencia con los objetivos de la secuencia. & 0,72 \\
\hline 3 & Pertinencia de la organización conceptual. & 0,76 \\
\hline 4 & Pertinencia de los conceptos. & 0,76 \\
\hline 5 & Pertinencia de las actividades. & 0,68 \\
\hline 6 & Pertinencia del lenguaje. & 0,64 \\
\hline \multirow[t]{2}{*}{7} & Pertinencia del uso de la herramienta GeoGebra. & 0,80 \\
\hline & Promedio. & 0,74 \\
\hline
\end{tabular}

Fuente. Elaboración propia

La secuencia didáctica se basó en cuatro misterios, los cuales fueron propuestos a los estudiantes para su trabajo. El primer misterio se muestra en la Figura 3. Éste se compone de tres momentos: el primero una invitación a desarrollar algunas acciones dentro del software de GeoGebra, el segundo unas preguntas que pretenden direccionar la actividad y el tercero, un reto en el que se invita a reflexionar y proponer una transformación propia del tema que se está estudiando.

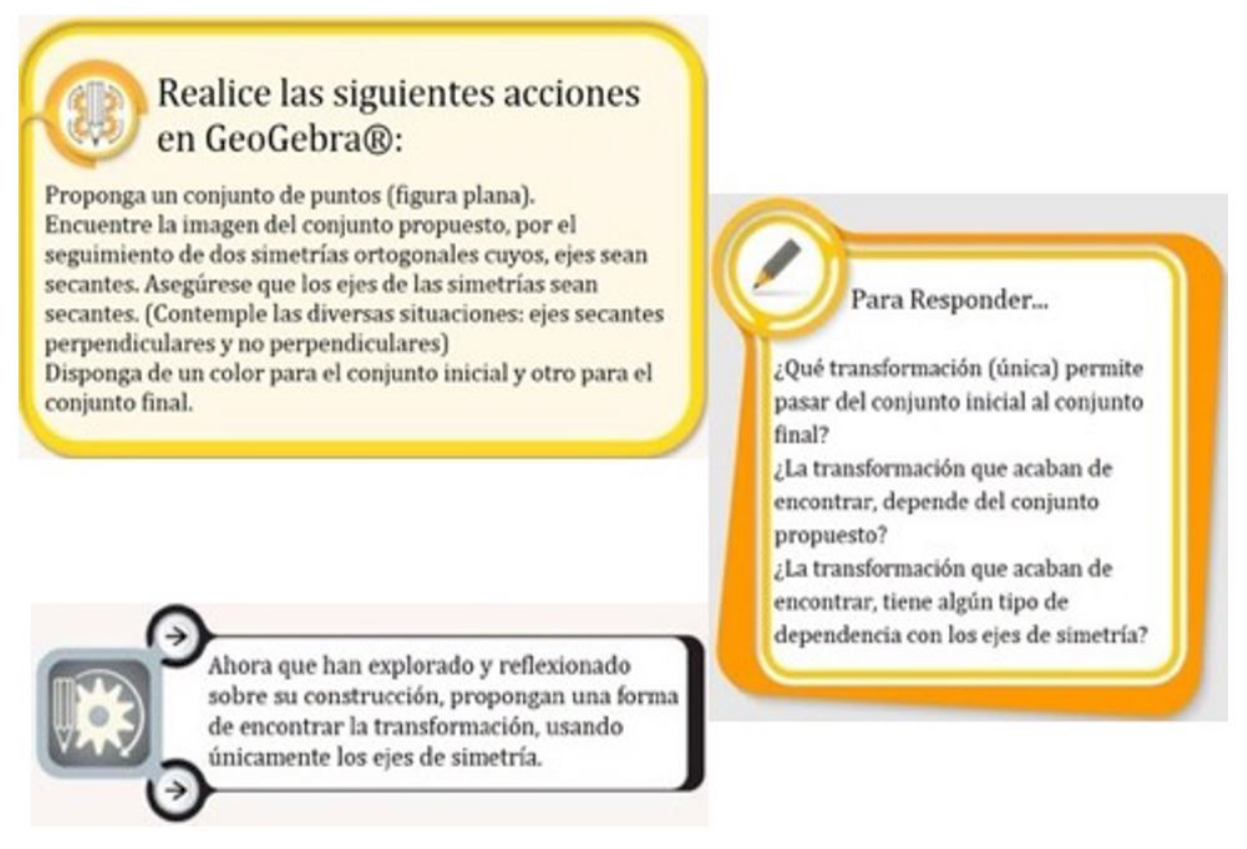

Figura 3. Primer misterio de la Secuencia Didáctica.

Fuente. Quezada-Alfonso y Robayo-Buitrago (2021)

\section{En relación con los aprendizajes}

En relación con el proyecto que implicó la secuencia didáctica, los estudiantes debían diseñar un teselado con base en los siguientes criterios: debe ser un diseño propio, se debe describir el proceso de construcción de la unidad de teselación, el diseño de la debe tener mínimo dos transformaciones y se debe identificar las transformaciones del plano teselado (Quezada-Alfonso \& Robayo-Buitago, 2021). 


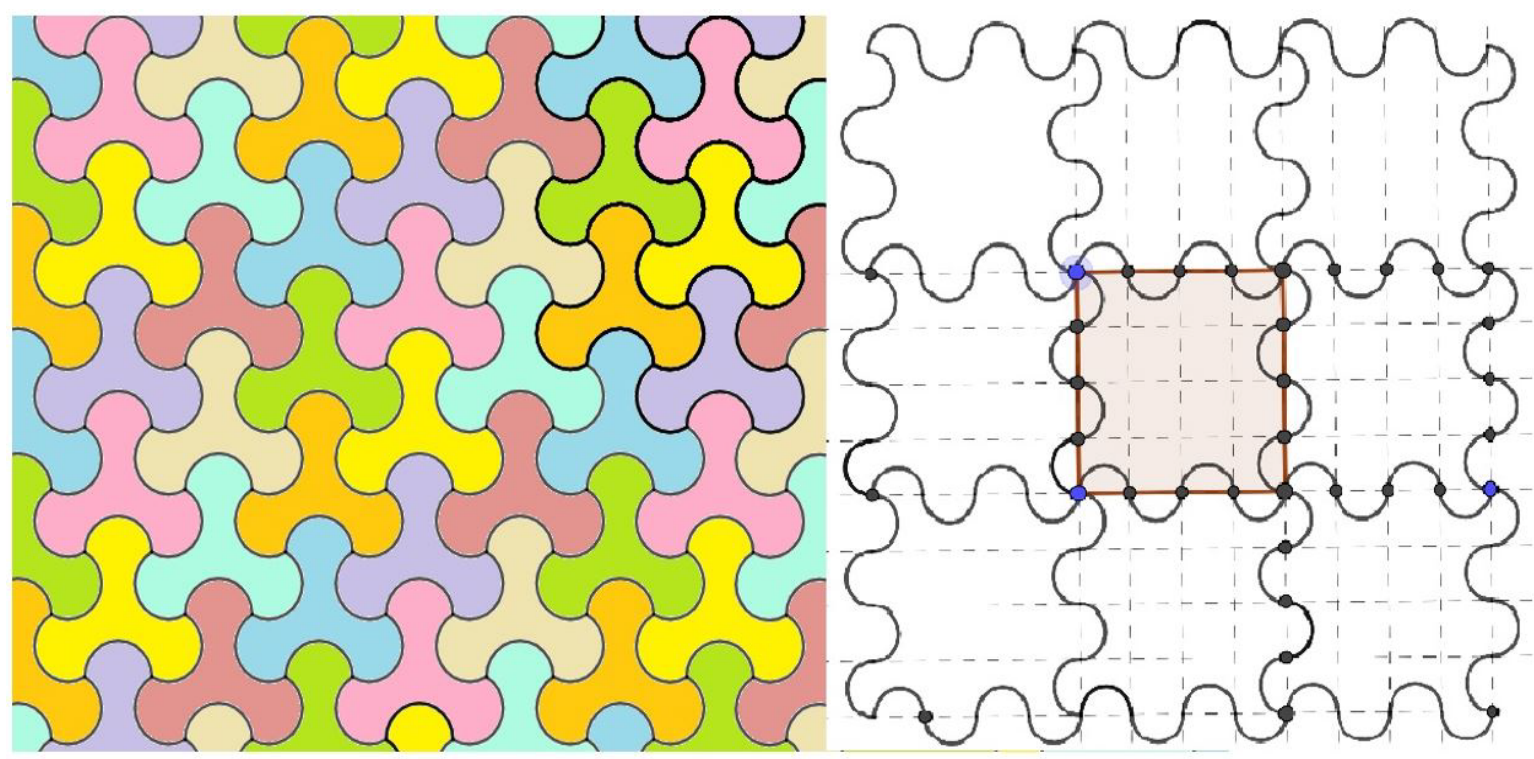

Figura 3. Teselado mediado con GeoGebra, resultado del proyecto de un grupo de estudiantes.

Fuente. Quezada-Alfonso y Robayo-Buitrago (2021)

La Figura 3 muestra uno de los teselados diseñados y desarrollados por un grupo de estudiantes que participaron de la investigación.

El Cuestionario utilizado en el postest tiene nueve preguntas y un solo contexto cuyo enunciado establece que "con base en la siguiente imagen responda las preguntas 1 a 9"; imagen que se observa en la Figura 4.

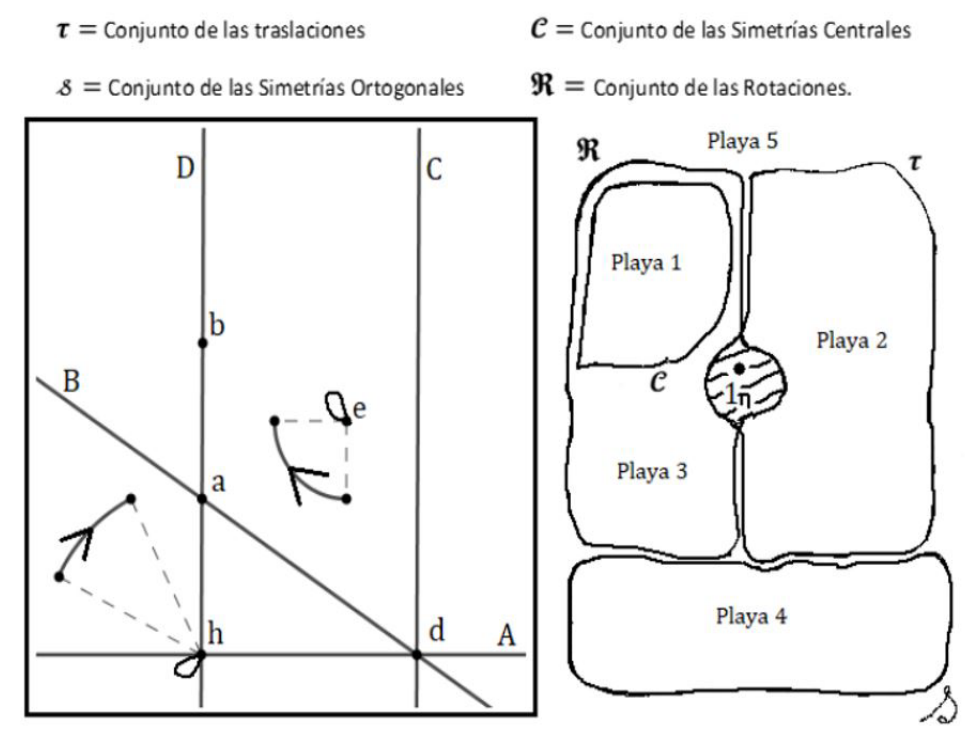

Figura 4. Contexto para las 9 preguntas del postest.

Fuente. Quezada-Alfonso y Robayo-Buitrago (2021) 
Las preguntas indagan por las transformaciones resultantes en el seguimiento de (1) $(a b) * S_{e},(2)(b a)$ ${ }^{*} r_{h}$, (3) $S_{a} * S_{e}$, (4) $S_{B}^{*} S_{C}$, (5) $S_{C} *(a b),(6) S_{C} * S_{D}$, (7) $S_{B}^{*} S_{d}$, (8) $S_{D}^{*} r_{h}$ y (9) $S_{a}^{*} S_{h}$. Las respuestas son de opción múltiple y se asumen en función de las playas 1 a 5 .

En la Figura 5, están sintetizados resultados de las diferentes pruebas de hipótesis que se realizaron para cada uno de los grupos G1 y G2 (Grupo ABPC y Grupo E. Habitual). Para cada caso, se verificaron los supuestos estadísticos necesarios.

El proceso para la aceptación de las hipótesis $(\mathrm{H} 1, \mathrm{H} 2, \mathrm{H} 3$ y $\mathrm{H} 4)$ se inicia determinando la normalidad de los datos obtenidos con la prueba Shapiro Wilk. Para aplicar la prueba de normalidad, las hipótesis $\mathrm{H} 1$ y $\mathrm{H} 2$ se apoyaron en la prueba de U-Mann Whitney, en tanto que las hipótesis H3 y H4 se apoyaron en la prueba de Wilcoxon.

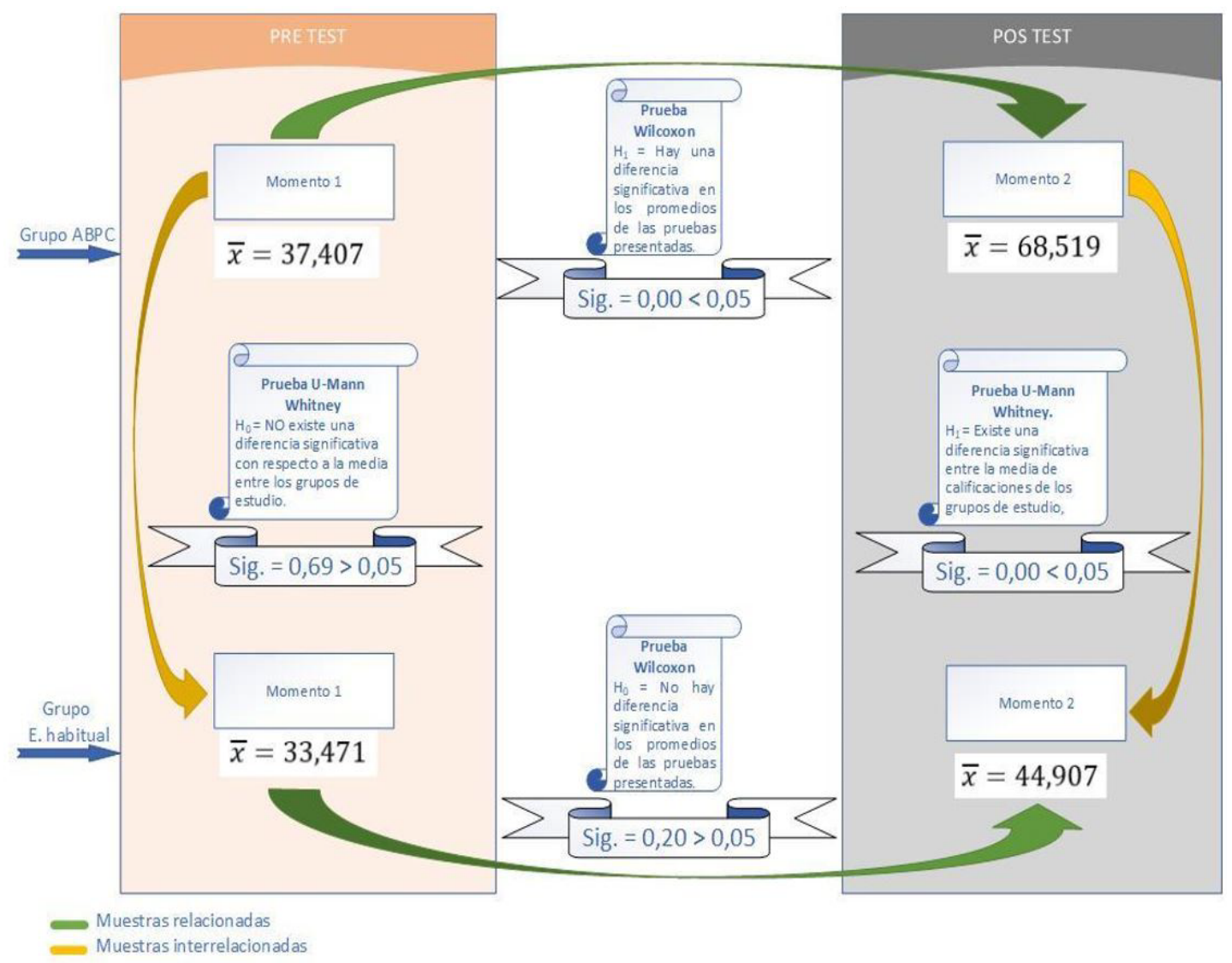

Figura 5. Resultados de las pruebas estadísticas aplicadas en el estudio.

Fuente. Elaboración propia con SPSS $20.0 \AA$ y Ms Excel®.

En cuanto a la Hipótesis H1 "El promedio de puntuaciones en el pre test del grupo G1 no tiene diferencias significativas con las obtenidas por el grupo G2", se acepta. Es decir, que el grupo G1 no tiene diferencias estadísticamente significativas en relación con el promedio de las puntuaciones. Este hecho, garantiza que los dos grupos son equiparables y que ninguno tiene ventajas respecto a otro, en relación con el aprendizaje de las simetrías axiales.

En relación con la hipótesis $\mathrm{H} 2$ "El promedio de las puntuaciones en la prueba pos test en los estudiantes del grupo G2 tiene diferencias significativas con las puntuaciones de estudiantes del grupo G1", se rechaza. Lo anterior, quiere decir 
que existen diferencias significativas en el promedio de puntuaciones entre los grupos G1 "Enseñanza habitual" y G2 "ABPC". Teniendo en cuenta que el grupo G2 obtuvo una media de 68.519 y que el grupo G1 obtuvo una media de 44.907, se infiere entonces que hay una mayor ganancia en el grupo G2, cuyo trabajo se desarrolló complementado con Aprendizaje Basado en Proyectos Colaborativos.

Con respecto de la Hipótesis H3 "No existe diferencia entre las puntuaciones pretest y postest para el grupo G1", se rechaza. Por tanto, se puede afirmar, que existe diferencia estadísticamente significativa entre las puntuaciones medias del pretest y el postest. Siendo la media del pretest 33,471 y la del postest 44,907, se puede inferir que hay una ganancia en el aprendizaje de las simetrías axiales en el grupo de grado octavo, cuya enseñanza aprendizaje se desarrolla en las condiciones habituales de la institución educativa.

Con base en los resultados obtenidos al evaluar la Hipótesis H4 "No Existe diferencia entre las puntuaciones pretest y postest para el grupo G2", se rechaza. Por lo anterior, es posible decir que existen diferencias estadísticamente significativas entre las puntuaciones de las medias del pretest y el postest. Considerando que la media del pretest es $37.407 \mathrm{y}$ que la media del poste es 68.519, se puede deducir que hay una ganancia en el aprendizaje de las simetrías axiales en el grupo de octavo grado, cuya enseñanza aprendizaje se desarrolla complementada con el Aprendizaje Basado en Proyectos Colaborativos.

\section{Conclusiones}

A la luz de los resultados expuestos en el apartado anterior, el éxito en las pruebas por parte del grupo ABPC, puede derivarse de la incidencia de la implementación y sus factores asociados: la modalidad de trabajo colaborativo, el enfoque del trabajo en proyectos y la relación con el mundo artístico (video y teselado; producto final del proyecto). Además, teniendo en cuenta que un grupo trabajó con las características del ABPC y el otro grupo con la enseñanza habitual; en el contexto de la nueva realidad, se encontró que las dos formas dan ganancias en el aprendizaje respecto al punto de partida. Los dos grupos sacaron provecho de la presencialidad remota, el uso y manejo de las TIC y el software de geometría dinámica GeoGebra, ampliando así, experiencias con este tipo de herramientas.

Adicionalmente, el trabajo con las características del ABPC que incorpora el uso del software GeoGebra, aporta elementos adicionales a la interacción entre los estudiantes, constituyendo un entorno favorable desde lo cognitivo, para la generación predictiva de las conjeturas geométricas que cada uno se plantea frente a la composición de simetrías axiales.

Los resultados obtenidos con todos los estudiantes concuerdan con los hallazgos que obtuvo Mariotti $(2003,2006)$ cuando utilizó Cabri ${ }^{\circledR}$ e identificó éste como un artefacto de mediación semiótica, válido en el contexto de la geometría. Sin embargo, se identifica como el ABPC da un valor agregado, desde el punto de vista cognitivo, en pro de identificar esta mediación para el aprendizaje de la geometría. Así las cosas, este trabajo se constituye en evidencia empírica que da indicios que permiten identificar el GeoGebra, como un artefacto de mediación tecnológico para el aprendizaje de la composición de simetrías axiales.

Indicar que la presente investigación tiene limitaciones que impiden generalizar sus resultados. En primera instancia se trabaja con una institución educativa con énfasis en matemáticas en la educación básica y media, no muchas instituciones tienen este énfasis en los primeros años de educación. La investigación se desarrolló en medio de la pandemia debido a la COVID-19, por lo que la modalidad podría decirse estuvo bajo un experimento educativo forzado. Sin embargo, esta investigación está portando evidencia empírica al indicar que la incorporación de la tecnología para la enseñanza 
aprendizaje de las matemáticas, potencialmente se constituye en una mediación, por lo que en futuras investigaciones es posible seguir avanzando en esta línea de indagación.

\section{Agradecimientos}

El equipo de investigación agradece al proyecto Curricular de la Maestría en Educación en Tecnología de la Universidad Distrital por el apoyo recibido en el desarrollo de la investigación. La investigación se enmarca en las líneas de acción del grupo de investigación DIDACTEC.

\section{Referencias}

Aiken, L. (1985). Three Coefficients For Analyzing The Reliability And Validity Of Ratings. Educational and Psychological Measurement, 45, 131-141. https://doi. org/10.1177/0013164485451012

Arbain, N., y Shukor, N. A. (2015). The effects of GeoGebra ${ }^{\circledR}$ on students achievement. ProcediaSocial and Behavioral Sciences, 172, 208-214

Badia, A. y García, C. (2006). Incorporación de las TIC en la enseñanza y el aprendizaje basados en la elaboración colaborativa de proyectos. Revista de Universidad y Sociedad del Conocimiento (RUSC). Vol. 3, n. ${ }^{\circ}$ 2. UOC. Recuperado de: http://www.uoc.edu/rusc/3/2/dt/esp/badia_ garcia.pdf

Bartolini-Bussi, M. G., y Mariotti, M. A. (2008). Semiotic mediation in the mathematics classroom: Artifacts and signs after a Vygotskian perspective (Trad. P. Perry). En L. English, M. G. Bartolini Bussi, G. Jones, R. Lesh y D. Tirosh (Eds.), Handbook of international research in mathematics education (pp. 746-783). Mahwah: Lawrence Erlbaum

Boluarte-Carbajal, A., y Tamari, K. (2017). Validez de contenido y confiabilidad inter-observadores de Escala Integral Calidad de Vida. Revista de Psicologia, 35(2), 641-666. https://doi. org/10.18800/psico.201702.009

Camargo, L., y Samper, C. (2013). Aproximación temprana al razonamiento geométrico en Educación Básica. In Simposio Nororiental de Matemáticas (Vol. 1, pp. 249-268)

Fajardo, M. D. C., y Larios, V. (2019). Descripción de procesos matemáticos en prácticas argumentativas. Educación Matemática, 31(3), $61-84$

García, S., y López, O. (2008). La enseñanza de la Geometría. En Colección: Materiales para apoyar la práctica educativa Coordinación (Primera Ed). Instituto Nacional para la Evaluación de la Educación. https://www.inee.edu.mx/wpcontent/uploads/2019/01/P1D401.pdf

Godino, J. y Ruíz, F. (2003). Geometría y su didáctica para maestros. Grupo Edumat, Universidad de Granada. España recuperado de https://www. ugr.es/ jgodino/edumat-maestros/

Gómez, C. L., y Ramirez, R. H. (2016). Comunidades de aprendizaje móvil, Mastery Learning y el problema 2 sigma como estrategias para el conocimiento previo en la resolución de actividades

Guba, E. G., y Lincoln, Y. S. (1994). Competing paradigms in qualitative research. In N. K. Denzin y Y. S. Lincoln (Eds.), Handbook of qualitative research (pp. 105-117). Thousand Oaks, CA: Sage

Hernández-Sampieri, R., Fernández-Collado, C., y Baptista-Lucio, M. (2014). Metodología de la investigación (J. Mares-Chacon (ed.)). McGraw Hill Intereamericana Editores S.A. http://www.casadellibro.com/librometodologia-de-la-investigacion-5-ed-incluyecd-rom/9786071502919/1960006

Iglesias-Inojosa, M., y Ortiz-Buitrago, J. (2019). La Demostración en Geometría desde una Perspectiva Didáctica. Revista Iberoamericana de Educación Matemática, 55, 159-183. http:// www.fisem.org/www/union/revistas/2019/55/ aula01.pdf

Maldonado, M. (2008). Aprendizaje basado en proyectos colaborativos. Una experiencia en educación superior. Laurus, Vol. 14, Núm. 28, pp. 158-180. Recuperado de: http:// 
redalyc.uaemex.mx/src/inicio/ArtPdfRed. jsp?iCve $=76111716009$

Mariotti, M. (2006). New artefacts and the mediation of mathematical meanings. Proceedings of the Seventeenth ICMI Study Conference "Technology Revisited". http://ims.mii.lt/ims/ konferenciju_medziaga/TechnologyRevisited/

Mariotti, M. A. (2009). Artifacts and signs after a Vygotskian perspective: the role of the teacher. ZDM, 41(4), 427-440

Martin-Romera, A., y Molina-Ruiz, E. (2017). Valor del conocimiento pedagógico para la docencia en Educación Secundaria: diseño y validación de un cuestionario. Estudios Pedagógicos, 43(2), 195-220. https://scielo.conicyt.cl/pdf/estped/ v43n2/art11.pdf

Moreno, L. (2014). Educación matemática: del signo al pixel. Bucaramanga: Universidad Industrial de Santander

McMillan, J. H., Schumacher, S., y Baides, J. S. (2005). Investigación educativa: una introducción conceptual. Madrid: Pearson

McMillan, J., y Schumacher, S. (2005). Investigación educativa. Editorial Pearson Educación S.A

Mesa, M. B., Morales, F. H. F., y DUARTE, J. E. (2018). Evaluación de una estrategia pedagógica basada en proyectos colaborativos mediados por TIC para el desarrollo de competencias en estadística

Ministerio de Educación Nacional (1998). Serie Lineamientos Curriculares. Recuperado de https://www.mineducacion.gov.co/1621/ articles-89869_archivo_pdf9.pdf

Morera, L., Fortuny, J. M., y Planas, N. (2012). Momentos clave en el aprendizaje de isometrías en un entorno colaborativo y tecnológico. Enseñanza de las ciencias: revista de investigación y experiencias didácticas, 143-154 Quezada-Alfonso, C., y Robayo-Buitrago, J. (2021). El Aprendizaje de la Composición de Simetrías Axiales, Bajo la Estrategia ABPC y el Uso de la Geometría Dinámica (GeoGebra $\left.{ }^{\circledR}\right)$ para Estudiantes de Grado Octavo del Colegio Refous. Universidad Distrital Francisco José de
Caldas

Rubio-Pizzorno, S., y Montiel-Espinosa, G. (2017). Consideraciones que epistémicas sobre los objetos geométricos en ambientes de geometría dinámica. Análisis inicial. En L. Serna (Ed.), Acta latinoamericana de matemática educativa (pp. 1505-1514). Comité Latinoamericano de Matemática Educativa

Sandoval-Cáceres, I. (2009). La geometría dinámica como una herramienta de mediación entre el conocimiento perceptivo y el geométrico. Educación matemática, 21(1), 5-27

Sua-Flórez, C., y Camargo-Uribe, L. (2019). Dynamic geometry and scientific reasoning: Duo to solve problems. Educacion Matematica, 31(1), 7-37. https://doi.org/10.24844/EM3101.01

Suárez, O., Molina-Vásquez, R., Orjuela, P., y Lizarazo, J. (2020). Elementos para la construcción de un modelo de tutor virtual. Editorial Fundación Universidad Autónoma de Colombia

Villamizar Araque, F. Y., Rincón Leal, O. L., y Vergel Ortega, M. (2018). Diseño de escenarios virtuales para problemas de optimización a través de geometría dinámica. Revista Logos Ciencia \& Tecnología, 10(2), 67-75. https://doi. org/10.22335/rlct.v10i2.571

Villamizar, F. Y. (2020). GeoGebra como herramienta mediadora de un fenómeno físico. Revista do Instituto GeoGebra Internacional de São Paulo. ISSN 2237-9657, 9(1), 76-89 